\section{Kidney \\ Blood Pressure Research}

Kidney Blood Press Res 2016;41:392-401

DOI: 10.1159/000443441

Published online: June 27, 2016

Accepted: May 10, 2016

\title{
Home- or Institutional Hemodialysis? - a Matched Pair-Cohort Study Comparing Survival and Some Modifiable Factors Related to Survival
}

\author{
Helena Rydella Naomi Clyne ${ }^{\mathrm{a}} \quad$ Mårten Segelmark $^{\mathrm{b}}$ \\ aDepartment of Nephrology Skåne University Hospital and Institution of Clinical Sciences Lund, Lund \\ University; ${ }^{b}$ Department of Nephrology and Department of Medical and Health Sciences, Linköping \\ University, Linköping, Sweden.
}

\section{Key Words}

Survival - Home hemodialysis - Hypertension - Volume control - Calcium and phosphate metabolism

\begin{abstract}
Background/Aims: Survival for dialysis patients is poor. Earlier studies have shown better survival in home-hemodialysis (HHD). The aims of this study are to compare survival for matched patients with HHD and institutional hemodialysis (IHD) and to elucidate the effect on factors related to survival such as hyperphosphatemia, fluid overload and anemia. Methods: In this retrospective, observational study, incident patients starting HHD and IHD were matched according to sex, age, comorbidity and date of start. Survival analysis was performed both as "intention to treat" including renal transplantation and "on treatment" with censoring at the date of transplantation. Dialysis doses, laboratory parameters and prescriptions of medications were compared. Results: After matching, 41 pairs of patients, with HHD and IHD, were included. Survival among HHD patients was longer compared with IHD, median survival being 17.3 and 13.0 years $(p=0.016)$, respectively. The "on treatment" analysis, also favoured HHD ( $p=0.015)$. HHD patients had lower phosphate, $1.5 \mathrm{mmol} / \mathrm{L}$ compared with 2.1 $\mathrm{mmol} / \mathrm{L}(\mathrm{p}<0.001)$ and no antihypertensives and diuretics compared with 2 for IHD patients at $6(p=0.001)$ and 18 months $(p=0.014)$. There were no differences in hemoglobin or albumin. Conclusion: HHD shows better survival compared with IHD, also after controlling for patient selection. This could be caused by better phosphate and/or fluid balance associated with higher dialysis doses.
\end{abstract}




\section{Kidney Blood Pressure Research}

\section{Introduction}

Despite improvement over time, survival for patients on maintenance dialysis is still poor. According to the Swedish Renal Registry (SRR), annual mortality for patients on dialysis was $30 \%$ in 1991 and $21 \%$ in 2012 [1]. Several studies have indicated a lower mortality for patients on home hemodialysis (HHD) [2-7]. We have previously reported an annual mortality of $4.9 \%$ for patients starting HHD in Lund during 1971-1998, with decreased mortality for each decade [8].

However, patients starting HHD are younger and healthier compared with patients starting on other dialysis modalities. It is still not clear whether there is a survival advantage related to HHD beyond patient selection, and if so the magnitude of the advantage and the cause of it.

A survival advantage for HHD beyond patient selection would most probably be mediated through improvement in risk factors for death common to all dialysis patients. Important characteristics of HHD are higher dialysis doses and more extensive patient education, which both have been linked to improved survival [9-11].

In order to elucidate these issues we conducted a retrospective, observational study with matched patients starting HHD and IHD as a randomized controlled trial is not feasible. The patient populations are collected from the HHD program at Lund University Hospital and from the IHD center at Malmö General Hospital in Sweden.

The main aim of this study was to compare survival in HHD patients with control IHD patients matched for sex, age, comorbidity and start period. A secondary aim was to study the effect of HHD as compared with IHD on factors related to survival such as subsequent renal transplantation, hyperphosphatemia, hypertension, anemia and hypoalbuminemia.

\section{Materials and Methods}

\section{Inclusion and matching criteria}

Incident patients starting HHD at Lund University Hospital from January $1^{\text {st }} 1983$ to December $31^{\text {st }}$ 2002 were eligible for the study if an appropriate IHD patient fulfilling the matching criteria listed below could be found among patients starting IHD at Malmö General Hospital. HHD patients were recruited from all over the Southern Health Care Region. An "incident patient" was defined as having less than 6 months with renal replacement therapy preceding start of HHD training.

Control incident IHD patients were required to have the same sex, the same level of comorbidity according to Davies Comorbidity Index [12], similar age ( $<5$ years difference) and similar start date of IHD ( $<5$ years difference). Davies Comorbidity Index is based on seven groups of comorbidities, malignancy, ischemic heart disease, peripheral vascular disease, left ventricular dysfunction, diabetes mellitus, systemic collagen vascular disease and other significant pathology with impact on survival in the general population. Patients with none of these comorbidities have grade 0 , those with 1-2 have grade 1 and those with 3 or more comorbidities have grade 2. As only patients completing HHD training were included in the study, patients receiving IHD for less than the median training period (72 days) were not accepted as matching controls in this study.

During the inclusion period, 118 patients started HHD training in Lund. Of these, 38 were not incident and 14 patients did not complete the training. Of these 14 patients, 6 changed to IHD, 5 received a renal transplant, 1 changed to PD and two were lost to follow-up after stopping HHD training and moving to other hospitals. Adequate matching patients with IHD were found for 41 of the remaining patients (Figure 1).

IHD patients were eligible for matching if they had started dialysis between January $1^{\text {st }} 1978$ and December $31^{\text {st }}$ 2007. During that period 377 patients started IHD at Malmö General Hospital. Every other HHD patient was matched with an IHD patient who had started within 5 years prior to or 5 years after the HHD patient's starting date. For each pair of patients matched according to start date and age, the remaining matching criteria were obtained from the patients' medical files. 


\section{Kidney Blood Pressure Research}

The majority of the HHD patients had their first dialysis at the HHD training clinic but 3 patients had PD, 9 had IHD and 2 patients had an unsuccessful renal transplant just before start of HHD. Among the IHD patients only one had a short period with PD before start of IHD.

\section{Collection of clinical data}

Date at start of dialysis, (i.e. start of HHD training or IHD) and information on switches to other renal replacement therapies were collected from patient files and the SRR. Survival data were collected from the Swedish Census. All other clinical data were collected from patient files.

Data on patient characteristics were collected at start of renal replacement therapy. Other clinical data were collected from start of HHD or IHD and until 18 months after start.

Weekly dialysis duration, frequency and blood flow were recorded at 6 and 18 months after start of HHD or IHD, respectively. Data on dialysis access were registered at start of HHD or IHD and at 18 months.

A time-averaged value of the levels of phosphate, calcium, hemoglobin and albumin in plasma/serum was calculated using all analyses performed during the period 6-18 months after start of HHD or IHD. The calculations were performed using an "area under the curve" approach and the number of data points varied between 1 and 60. For patients stopping dialysis before 18 months (transplantation/death/change to another dialysis modality), only data up to the date of such an event was used for the calculations.

The number of prescribed phosphate binders was recorded according to Anatomic Therapeutic Chemical Classification, ATC-codes, according to WHO [13], at 6 and 18 months after start of HHD or IHD. Prescriptions of anti-hypertensive drugs and diuretics were recorded as Defined Daily Doses (DDD), according to WHO [14] and as number of drugs according to ATC codes at 6 and 18 months. Prescriptions of erythropoiesis stimulating agents (ESA) were recorded as DDD at 6 and 18 months.

For HHD patients, blood pressure at the visit to the clinic in closest proximity to 6 and 18 months after start of HHD was recorded. For IHD patients, mean pre-dialysis blood pressure during the $6^{\text {th }}$ and $18^{\text {th }}$ month after start of IHD was recorded.

All patients were followed until death or January $1^{\text {st }} 2013$. No patient was lost to follow-up.

\section{Statistical Analysis}

Statistical analyses were performed with IBM SPSS version 20 and GraphPad Prism 6.

For survival analysis Kaplan Meier curves and Breslow test were used. All patients were included in the survival analysis. Survival analysis was performed as intention to treat analysis, where patients were considered at risk also after changes to other modalities of renal replacement therapy, including transplantation. To remove the impact of differences between the groups after subsequent renal transplantation survival analysis was also performed as on treatment analysis, where patients were censored as lost to follow up on the day of renal transplantation but not when switching to another dialysis modality.

Fig. 1. Flow-chart showing selection of HHD patients. Of 118 patients starting HHD in Lund during the study period dent. Of the remaining patients, 14 did not complete the training. 66 patients met the inclusion criteria but for 25 of these no adequate matching IHD patient was found.

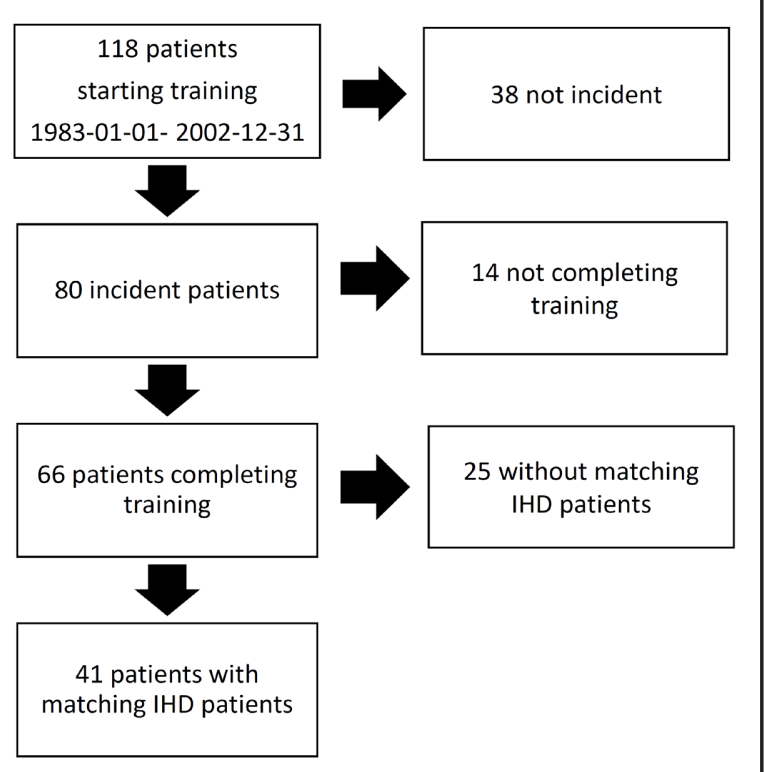




\section{Kidney Blood Pressure Research}

Table 1. Patient characteristics at start of renal replacement therapy and duration and frequency of renal replacement therapies

\begin{tabular}{lcc}
\hline & HHD & IHD \\
\hline $\begin{array}{l}\text { Patients number } \\
\text { Median age (range) years }\end{array}$ & $41.5(19.4-71.3)$ & $53.9(18.9-74.0)$ \\
Females & $10(24 \%)$ & $10(24 \%)$ \\
Davies comorbidity grade & & \\
- $\quad 0$ & $29(71 \%)$ & $29(71 \%)$ \\
- 1 & $12(29 \%)$ & $12(29 \%)$ \\
- 2 & $0(0 \%)$ & $0(0 \%)$ \\
Renal diagnosis & & \\
- Glomerulonephritis & $23(56 \%)$ & $18(44 \%)$ \\
- Adult polycystic kidney disease & $11(27 \%)$ & $9(22 \%)$ \\
- Diabetes nephropathy & $1(2.4 \%)$ & $5(12 \%)$ \\
- Nefrosclerosis & $1(2.4 \%)$ & $2(5 \%)$ \\
- Other & $5(12 \%)$ & $2(5 \%)$ \\
- Unknown & 0 & $1.7(0.2-27.0 ; \mathrm{n}=41)$ \\
Median duration (Range) Years & & $0(\mathrm{n}=0)$ \\
- First period with HHD/IHD & $1.8(0.5-12.2 ; \mathrm{n}=41)$ & $1.8(0.2-27.0 ; \mathrm{n}=41)$ \\
- Total treatment with HHD & $2,7(0.5-24.9 ; \mathrm{n}=41)$ & $11.7(0.05-27.1 ; \mathrm{n}=23)$ \\
- Total treatment with IHD & $0.6(0.02-19.0 ; \mathrm{n}=26)$ & $10.8(0.21-28.4 ; \mathrm{n}=41)$ \\
\hline$\quad$ Renal transplantation & $10.6(0.02-28 ; \mathrm{n}=33)$ & \\
- Follow-up until January 1st 2013 & $14.2(4.3-29.9 ; \mathrm{n}=41)$ & \\
\hline
\end{tabular}

Only matched pairs with complete values were included in other comparisons between groups and paired tests were used. Paired t-test was used for laboratory parameters. Mc Nemar was used for dialysis access and Wilcoxon matched-pairs signed rank test was used for dialysis prescriptions and medications. Descriptive data are given as mean and standard deviation or median and range (minimum and maximum).

As blood tests are not taken with equal intervals, I e every week or month, the "area under the curve" approach was used for laboratory parameters. This is a better measure of the patients mean values compared with just the plain mean. For example values of hemoglobin taken every day when a patient has a gastrointestinal bleeding will have the same impact on the mean value as hemoglobin values taken monthly when the patient is not bleeding.

Ethics

The study was approved by the Regional Ethical Review Board in Lund.

\section{Results}

Patient characteristics and renal replacement therapies during the study

A total of 41 matched pairs of HHD and IHD patients were included in the study. Median age for included HHD and IHD patients was 51.5 and 53.9 years, respectively, and $24 \%$ of all patients were female. Most pairs had a Davies Comorbidity Index of 0 (71\%), none had an index higher than 1 . Davies Comorbidity Index grade 1 represents 1-2 comorbidities. The most common comorbidity for HHD patients was systemic collagen vascular disease (12 $\%$ ), followed by diabetes mellitus (5\%).Only one of the HHD patient had 2 comorbidities, systemic collagen vascular disease and chronic obstructive lung disease. Out of the patients with IHD and Davies Comorbidity grade 1,15 \% had 2 comorbidities. Diabetes was the most common comorbidity (17\%), followed by peripheral vascular disease $(7 \%)$, ischaemic heart disease (7 \%) and systemic collagen disease (5\%). For both HHD and IHD patients, glomerulonephritis was the most common renal diagnosis followed by adult polycystic kidney disease (Table 1).

Median follow up duration was 14 (range 4-30) years for HHD patients and 11 (range $0.2-28$ ) years for IHD patients. In both treatment groups a majority of patients changed renal replacement therapy after start of HHD or IHD. Only one patient in each group continued their initial treatment modality until the end of follow-up, while 2 HHD patients and 15 


\section{Kidney \\ Blood Pressure Research}

IHD patients died without change in therapy. At 6 months after start, no HHD patient but 7 IHD patients had switched to other dialysis modalities or received a renal transplant. At 18 months 16 HHD and 19 IHD patients had switched renal replacement therapy. Some patients had more than one period with HHD or IHD. The median duration of the first period of HHD and IHD, was 1.8 and 1.7 years, respectively (Table 1).

\section{Survival}

All patients were followed from start of renal replacement therapy until January $1^{\text {st }} 2013$. At that date 16 (39\%) HHD patients and 11 (27\%) IHD patients were still alive. In the Kaplan Meier analysis, survival for HHD patients was significantly longer compared with IHD patients $(\mathrm{p}=0.016)$. Mean survival for HHD patients was 17.3 years compared with 13.0 for the IHD patients (median 16.7 and 11.2 years respectively). Five-year survival was $98 \%$ for HHD patients (n at risk 41 ) and $71 \%$ for IHD patients (n at risk 30 ) and the corresponding figures for ten-year survival was $73 \%$ (n at risk 31 ) compared with $56 \%$ (n at risk 24; Figure 2).

\section{Renal transplantation}

After starting HHD or IHD, 33 HHD and 23 IHD patients, respectively, underwent a renal transplantation. Of these, 5 HHD patients and 4 IHD patients, did get a transplant from a living donor. If treating transplantation as "lost to follow up", i.e. as censured in survival analysis, the contribution of renal transplantation to survival is removed. In such "on treatment" analysis HHD patients still exhibited a significantly longer survival $(p=0.015)$. Six of the HHD patients died before censuring compared with 17 of the IHD patients. The "on-treatment" 5 years survival, that is excluding time after renal transplantation, was $91 \%$ for HHD patients and $67 \%$ for IHD patients. After 10 years, only 4 HHD and 7 IHD remained at risk.

\section{Dialysis dose and dialysis access}

Median weekly duration of the dialysis sessions was significantly longer for HHD compared with IHD patients, both at $6(\mathrm{p}<0.001)$ and 18 months after start $(\mathrm{p}=0.001)$. At 6 months the median weekly duration was 15 hours (range 10.5-38.5) for HHD patients and 12 hours (range 8-15) for IHD patients. At 18 months the median weekly duration was 15.5 hours (range 12-28) for HHD patients and 12 hours (range 8-15) for IHD patients (Table 2).

HHD patients also had a significantly higher frequency of dialysis sessions per week at 6 months $(p=0.005)$, but not at 18 months $(p=0.066)$. The mean blood flow at 6 months was higher for IHD compared with HHD patients $(\mathrm{p}=0.040)$, but at 18 months the difference no longer showed statistical significance $(\mathrm{p}=0.14)$ (Table 2). tively. Long time survival for matched pairs of home-hemodialysis pa$(\mathrm{p}=0.016$ Breslow). Mean survival for HHD and IHD patients was 17.3 and 13.0 years, respectively. At ten years after start of renal replacement therapy 31 HHD patients and 24 IHD patients were still at risk. At twenty years 8 HHD patients and 7 IHD patients were at risk. 


\section{Kidney Blood Pressure Research}

Table 2. Dialysis doses at 6 and 18 months after start of HHD and IHD

\begin{tabular}{|c|c|c|c|c|c|c|c|c|}
\hline & \multicolumn{2}{|c|}{ HHD } & \multicolumn{2}{|c|}{ IHD } & \multicolumn{2}{|c|}{$\mathrm{p}$ value } & \multicolumn{2}{|c|}{$\mathrm{n}$} \\
\hline Months after start & 6 & 18 & 6 & 18 & 6 & 18 & 6 & 18 \\
\hline Dialysis duration per week & 15 & 15.5 & 12 & 12 & $<0.001$ & 0.001 & 28 & 14 \\
\hline$h$ & $(10.5-38.5)$ & $(12-28)$ & $(8-15)$ & $(8-15)$ & & & & \\
\hline Dialysis frequency per week & $\begin{array}{c}3 \\
(3-6)\end{array}$ & $\begin{array}{c}3 \\
(3-4)\end{array}$ & $\begin{array}{c}3 \\
(2-3)\end{array}$ & $\begin{array}{c}3 \\
(3-3)\end{array}$ & 0.005 & 0.066 & 28 & 14 \\
\hline $\begin{array}{l}\text { Blood flow } \\
\mathrm{ml} / \mathrm{min}\end{array}$ & $\begin{array}{c}240 \\
(200-325)\end{array}$ & $\begin{array}{c}250 \\
(200-325)\end{array}$ & $\begin{array}{l}250 \\
(225-400)\end{array}$ & $\begin{array}{c}300 \\
(250-400)\end{array}$ & 0.040 & 0.14 & 13 & 7 \\
\hline
\end{tabular}

At start of HHD $76 \%$ of the HHD patients had an AV fistula or graft compared with $46 \%$ of the IHD patients $(\mathrm{p}=0.008)$. At 18 months after start of HHD or IHD (or at switch to another dialysis modality or renal transplantation before 18 months), 93\% compared with $76 \%$ patients had an AV fistula or graft $(p=0.39)$.

\section{Calcium and phosphate balance \\ Mean time-averaged plasma} phosphate levels were significantly lower for HHD patients, 1.5 mmol/l, compared with IHD between HHD and IHD patients $(p=0.33)$ (Table 3$)$. There was no difference in the number between HHD and IHD patients ( $\mathrm{p}=0.33$ ) (Table 3$)$. There was no difference in the number of prescribed phosphate binders according to ATC codes at 6 and 18 months, respectively, between HHD and IHD patients (21 pairs; $p=0.74,11$ pairs; $p>0.99$ ). Both groups had one prescribed phosphate binder at 6 and 18 months.

\section{Blood pressure and fluid balance}

Mean blood pressure at 6 months was 141/81 mmHg for HHD patients and 151/86 for IHD patients. At 18 months mean blood pressure was 149/82 mmHg and 150/84, respectively. As recording of blood pressure was not standardized and performed under different circumstances, we did not conduct a statistical analysis.

IHD patients had a significantly higher number of antihypertensive drugs (including diuretics) based on ATC codes both at $6(p<0.001)$ and at $18(\mathrm{p}=0.014)$ months after start of HHD or IHD (Table 4). At 6 and 18 months, 23 (56 \%) and 16 (64\%) HHD patients, respectively, had no antihypertensive medication compared with 2 (6\%) and 1 (5\%) IHD patients.

The number of antihypertensive drugs based on ATC codes not including diuretics was significantly higher at $6(\mathrm{p}=0.002)$ and 18 months $(\mathrm{p}=0.037)$ for IHD patients. Based on DDD, the differences in antihypertensive drugs were not significant at $6(\mathrm{p}=0.11)$ or 18 months $(\mathrm{p}=0.21)$ (Table 4).

IHD patients had significantly more diuretics based on DDD at $6(p=0.007)$ and 18 $(p=0.043)$ months. For IHD patients the median DDD was 6.3 and 3.1 at 6 and 18 months, respectively. For HHD patients the median DDD was 0 at 6 and 18 months (Table 4). 


\section{Kidney Blood Pressure Research}

Table 4. Median number of antihypertensives and diuretics according to Anatomic Therapeutic Chemical Classification (ATC codes) and Defined Daily Doses (DDD) according to WHO at 6 and 18 months after start of HHD or IHD

\begin{tabular}{lcccccccc}
\hline & \multicolumn{2}{c}{ HHD } & \multicolumn{2}{c}{ IHD } & \multicolumn{2}{c}{ P value } & \multicolumn{2}{c}{$\mathrm{n}$} \\
\hline Months after start & 6 & 18 & 6 & 18 & 6 & 18 & 6 & 18 \\
Number of antihyper- & 0 & 0 & 2 & 2 & $<0.001$ & 0.014 & 26 & 10 \\
tensives and diuretics & $(0-3)$ & $(0-3)$ & $(0-5)$ & $(0-5)$ & & & & \\
Number of antihyper- & 0 & 0 & 2 & 2 & 0.002 & 0.037 & 26 & 10 \\
tensives only & $(0-2)$ & $(0-3)$ & $(0-4)$ & $(0-4)$ & & & & \\
DDD antihypertensives & 0 & 0 & 1,3 & 1,2 & 0.11 & 0.21 & 24 & 9 \\
& $(0-4)$ & $(0-2,4)$ & $(0-5.2)$ & $(0-5.67)$ & & & & \\
DDD diuretics & 0 & 0 & 6.3 & 3.13 & 0.007 & 0.043 & 26 & 10 \\
& $(0-13)$ & $(0-13)$ & $(0-25)$ & $(0-25)$ & & & & \\
\hline
\end{tabular}

7 IHD patients had changed renal replacement therapy at 6 months after start. 16 HHD and 19 IHD patients had changed at 18 months after start. Only pairs of patients (n) still on HHD or IHD and with complete records for each prescription or dose were included in the analysis.

\section{Renal anemia and plasma albumin}

There were no significant differences in the time averaged blood hemoglobin concentration during the period $6-18$ months although there was a trend towards higher mean levels for the HHD patients, $106 \mathrm{~g} / \mathrm{L}$ compared with $102 \mathrm{~g} / \mathrm{L}(\mathrm{p}=0.33$ ) (Table 3). There were no significant differences in prescribed doses of ESA per week at 6 months after start of HHD or IHD (0.078). However, at 18 months the HHD patients had significantly higher doses $(p=0.038), 7700$ IE per week compared with 3010 IE per week for the IHD patients.

There was no significant difference in plasma-albumin between HHD and IHD patients $(\mathrm{p}=0.56)$ (Table 3).

\section{Discussion}

In this retrospective observational case-control study we found a significant survival advantage for HHD compared with IHD. Patients with HHD lived on average four years longer compared with IHD patients. This finding is in accordance with other retrospective observational studies, comparing dialysis at home with dialysis at an institution [4-7]. However few of the earlier studies include matching for both age, comorbidity index and start date. As randomized controlled studies are difficult and blinded studies impossible to perform in this field, well matched observational case control studies are an attractive approach for the comparison of dialysis modalities. Malmö General Hospital is located in the region of recruitment for the HHD program in Lund, but as very few patients were actually referred from Malmö to the program, IHD patients from Malmö constitute an appropriate control group.

Improved survival for patients with HHD compared with matched patients with IHD has been shown by Saner et al and by Weinhandl et al, but none of these studies have matched for both age and a validated comorbidity index [4, 7]. Other studies have taken age or comorbidity into account through multivariate analysis $[4,6,15]$. In one study, patients receiving hemodialysis at long-term care facilities such as nursing homes were defined as having HHD [15]. Albeit, that they did not perform the dialysis themselves or control dialysis dose. Multivariate analysis was used showing that survival for this form of HHD was inferior to IHD. This highlights the importance of a uniform definition of HHD versus IHD as well as that matching seems to be superior to multivariate analysis when comparing survival between dialysis modalities. Finally, in an earlier study we found that age, comorbidity index and start date of dialysis all had a major impact on survival in HHD patients [8]. Subsequent renal transplantation, has most probably contributed to survival in both groups, but was 
more common in the HHD patients compared with the IHD patients in our study. By treating patients as lost to follow up after receiving a renal transplant in an "on-treatment" analysis, we can deduce that the survival advantage is not only an effect of higher frequency of renal transplantation. This is consistent with our earlier study, where we did not find a statistically significant contribution of transplantation in patients starting HHD [8]. Pauly et al has reported that survival for patients with renal transplants from deceased donors does not differ from survival in HHD patients. However, survival for patients with renal transplants from living donors was superior compared with patients on HHD [3].

The main clinical differences between the modalities are that HHD patients receive more education, are responsible for their treatment and thus accept higher dialysis doses. Devins et al has shown an association between patient education and survival for patients with chronic kidney disease [11]. The median HHD training period for the patients in Lund was 72 days and consisted of an individual but standardized educational program conducted by a specialized nurse on a one to one basis. Patients were also given freedom to increase their dialysis dose and adapt timing to their everyday lives.

The dialysis dose in this study, defined as weekly duration and frequency, was recorded at 6 and 18 months after start. Median weekly duration was longer for HHD patients compared with IHD patients both at 6 and 18 months. Interestingly, the difference in weekly duration was mainly an effect of session length. The duration and frequency, 12 hours and thrice weekly, for the IHD patients corresponds to the so-called conventional hemodialysis as described in many earlier reports studying effects of different dialysis doses [16-19]. A number of studies describe improved survival related to both prolonged session length and increased frequency of sessions $[9,10,20]$. The association between duration of sessions and mortality is shown to be unrelated to levels of Kt/V [10] indicating that factors other than the clearance of small molecules are related to mortality.

Clearance of phosphate is one such factor that differs significantly from small molecule clearance because of an aqueous cover, binding to different salts and slow transportation from the intracellular to the extracellular space [21]. Several earlier studies have shown improved plasma phosphate levels related to increased dialysis dose compared with conventional hemodialyis $[16,17,22]$. The HHD patients in our study had significantly lower plasma phosphate levels, defined as time averaged mean 6-18 months after start, compared with IHD patients. We consider 6-18 months after start to be an adequate proxy for time spent on respective dialysis modality, as well as leaving a sufficient number of patients left in treatment. Differences in plasma phosphate levels are not only related to dialysis dose, but also to dietary phosphate intake and to number and actual intake of prescribed phosphate binders. Dietary phosphate intake was not known in this study but prescription of phosphate binders was recorded. Interestingly, the number of phosphate binders according to ATC codes was the same for both treatment groups. Increased dialysis dose, increased understanding of and compliance to prescribed phosphate binders and greater adherence to dietary advice are possible explanations for the lower plasma phosphate levels in the HHD patients.

Fluid balance, blood pressure and left ventricular hypertrophy are other factors that are related to survival $[23,24]$ and improved by a higher dialysis dose [16, 17, 22, 25]. Some earlier studies have described associations between increased dialysis dose and decreased blood pressure and prescription of antihypertensive drugs [16, 22, 25]. In one of these studies, by Nesrallah et al, interdialytic weight gains were also studied and shown to be decreased with increased dialysis dose [25]. In our study the median number of antihypertensive drugs, with or without diuretics, was lower both at 6 and 18 months for HHD patients. The majority of HHD patients had no antihypertensive drugs or diuretics at either 6 or 18 months. Based on DDD, there were no significant differences related to antihypertensive drugs, which most probably is a reflection of the fact that DDD is defined for a population without renal failure and not applicable to patients with end-stage renal disease. The lower number of prescribed antihypertensive drugs and diuretics for HHD patients might be related to a more gentle ultrafiltration process, due to higher dialysis dose and better compliance to both prescribed 


\section{Kidney Blood Pressure Research}

dry weight and prescribed fluid restrictions, which in turn results in less thirst and lower interdialystic weight gain.

Renal anemia is also related to survival in dialysis patients [26], but previous studies show divergent results concerning the influence of different dialysis doses [16, 17, 22]. In our study, there was a trend towards a higher hemoglobin concentration for the HHD patients. The difference might to some extent be explained by higher doses of ESA. The reason for differences in doses is not known. To our knowledge, there was no difference in target values for hemoglobin. One possible explanation is the trend that HHD patients had more frequent dialysis, which might be related to more frequent small blood losses and secondary to that higher ESA doses to maintain the hemoglobin level. It is also possible that the IHD patients received intravenous iron more frequently than the HHD patients. The average plasma levels of albumin were similar and normal in both treatment groups, thus ruling out different degrees of inflammation in the HHD and IHD patients, respectively.

Because of the retrospective design, there are limitations to this study. The most important is that despite our strict matching, there is still a risk of differences between HHD and IHD patients not controlled for. On the other hand, due to the strict matching, few patients could be included in the study. As to the factors related to survival, differences in prescriptions of phosphate binders could only be compared as number of and not as the phosphate binding index developed by Daugirdas et al [27]. Only surrogate measures, i.e. prescriptions of antihypertensive drugs and diuretics, were available, for studying fluid balance. Prescriptions of iron were missing.

\section{Conclusion}

In conclusion this study shows a significant survival advantage for HHD patients compared with IHD patients irrespective of age, comorbidity, start date and subsequent renal transplantation. HHD patients had a significantly higher dialysis dose, lower levels of plasma phosphate and were prescribed fewer antihypertensive drugs and diuretics, despite similar blood pressure, compared with IHD patients. We believe that these factors independently and together contribute to the superior survival of the HHD patients.

\section{Disclosure Statement}

The authors have no conflicts of interest.

\section{Acknowledgments}

Helena Rydell has received grants from Skåne Regional Council, The Southern Health Care Region in Sweden, Paul Frankenius Foundation and Swedish Society of Nephrology.

\section{References}

1 Swedish Renal Registry Annual Report 2013

2 Grant AC, Rodger RS, Howie CA, Junor BJ, Briggs JD, Macdougall AI: Dialysis at home in the west of Scotland: a comparison of hemodialysis and continuous ambulatory peritoneal dialysis in age- and sex-matched controls. Perit Dial Int 1992;12:365-368.

3 Pauly RP, Gill JS, Rose CL, Asad RA, Chery A, Pierratos A, Chan CT: Survival among nocturnal home haemodialysis patients compared to kidney transplant recipients. Nephrol Dial Transplant 2009;24:2915-2919.

4 Saner E, Nitsch D, Descoeudres C, Frey FJ, Uehlinger DE: Outcome of home haemodialysis patients: a casecohort study. Nephrol Dial Transplant 2005;20:604-610. 


\section{Kidney \\ Blood Pressure Research}

Rydell et al:: Survival on Home Hemodialysis

5 Nitsch D, Steenkamp R, Tomson CR, Roderick P, Ansell D, MacGregor MS: Outcomes in patients on home haemodialysis in England and Wales, 1997-2005: a comparative cohort analysis. Nephrol Dial Transplant 2011;26:1670-1677.

6 Marshall MR, Hawley CM, Kerr PG, Polkinghorne KR, Marshall RJ, Agar JW, McDonald SP: Home hemodialysis and mortality risk in Australian and New Zealand populations. Am J Kidney Dis 2011;58:782793.

7 Weinhandl ED, Liu J, Gilbertson DT, Arneson TJ, Collins AJ: Survival in daily home hemodialysis and matched thrice-weekly in-center hemodialysis patients. J Am Soc Nephrol 2012;23:895-904.

8 Rydell H, Krutzen L, Simonsen O, Clyne N, Segelmark M: Excellent long time survival for Swedish patients starting home-hemodialysis with and without subsequent renal transplantations. Hemodial Int 2013;17:523-531.

9 Flythe JE, Curhan GC, Brunelli SM: Shorter length dialysis sessions are associated with increased mortality, independent of body weight. Kidney Int 2013;83:104-113.

10 Saran R, Bragg-Gresham JL, Levin NW, Twardowski ZJ, Wizemann V, Saito A, Kimata N, Gillespie BW, Combe C, Bommer J, Akiba T, Mapes DL, Young EW, Port FK: Longer treatment time and slower ultrafiltration in hemodialysis: associations with reduced mortality in the DOPPS. Kidney Int 2006;69:1222-1228.

11 Devins GM, Mendelssohn DC, Barre PE, Taub K, Binik YM: Predialysis psychoeducational intervention extends survival in CKD: a 20-year follow-up. Am J Kidney Dis 2005;46:1088-1098.

12 Davies SJ, Phillips L, Naish PF, Russell GI: Quantifying comorbidity in peritoneal dialysis patients and its relationship to other predictors of survival. Nephrol Dial Transplant 2002;17:1085-1092.

13 http://www.whocc.no/atc/structure_and_principles/:

14 http://www.whocc.no/ddd/definition_and_general_considera/:

15 MacRae JM, Rose CL, Jaber BL, Gill JS: Utilization and outcome of 'out-of-center hemodialysis' in the United States: a contemporary analysis. Nephron Clin Pract 2010;116:c53-59.

16 Rocco MV, Lockridge RS, Jr., Beck GJ, Eggers PW, Gassman JJ, Greene T, Larive B, Chan CT, Chertow GM, Copland M, Hoy CD, Lindsay RM, Levin NW, Ornt DB, Pierratos A, Pipkin MF, Rajagopalan S, et al. : The effects of frequent nocturnal home hemodialysis: the Frequent Hemodialysis Network Nocturnal Trial. Kidney Int 2011;80:1080-1091.

17 Ayus JC, Mizani MR, Achinger SG, Thadhani R, Go AS, Lee S: Effects of short daily versus conventional hemodialysis on left ventricular hypertrophy and inflammatory markers: a prospective, controlled study. J Am Soc Nephrol 2005;16:2778-2788.

18 Daugirdas JT, Chertow GM, Larive B, Pierratos A, Greene T, Ayus JC, Kendrick CA, James SH, Miller BW, Schulman G, Salusky IB, Kliger AS, Frequent Hemodialysis Network Trial G: Effects of frequent hemodialysis on measures of CKD mineral and bone disorder. J Am Soc Nephrol 2012;23:727-738.

19 Mucsi I, Hercz G, Uldall R, Ouwendyk M, Francoeur R, Pierratos A: Control of serum phosphate without any phosphate binders in patients treated with nocturnal hemodialysis. Kidney Int 1998;53:1399-1404.

20 Group FHNT, Chertow GM, Levin NW, Beck GJ, Depner TA, Eggers PW, Gassman JJ, Gorodetskaya I, Greene T, James S, Larive B, Lindsay RM, Mehta RL, Miller B, Ornt DB, Rajagopalan S, Rastogi A, Rocco MV, Schiller B, Sergeyeva O, Schulman G, Ting GO, Unruh ML, Star RA, Kliger AS: In-center hemodialysis six times per week versus three times per week. N Engl J Med 2010;363:2287-2300.

21 Kuhlmann MK: Phosphate elimination in modalities of hemodialysis and peritoneal dialysis. Blood Purif 2010;29:137-144.

22 Culleton BF, Walsh M, Klarenbach SW, Mortis G, Scott-Douglas N, Quinn RR, Tonelli M, Donnelly S, Friedrich MG, Kumar A, Mahallati H, Hemmelgarn BR, Manns BJ: Effect of frequent nocturnal hemodialysis vs conventional hemodialysis on left ventricular mass and quality of life: a randomized controlled trial. JAMA 2007;298:1291-1299.

23 Stack AG, Saran R: Clinical correlates and mortality impact of left ventricular hypertrophy among new ESRD patients in the United States. Am J Kidney Dis 2002;40:1202-1210.

24 Chang TI, Flythe JE, Brunelli SM, Muntner P, Greene T, Cheung AK, Chertow GM: Visit-to-visit systolic blood pressure variability and outcomes in hemodialysis. J Hum Hypertens 2014;28:18-24.

25 Nesrallah G, Suri R, Moist L, Kortas C, Lindsay RM: Volume control and blood pressure management in patients undergoing quotidian hemodialysis. Am J Kidney Dis 2003;42:13-17.

26 Volkova N, Arab L: Evidence-based systematic literature review of hemoglobin/hematocrit and all-cause mortality in dialysis patients. Am J Kidney Dis 2006;47:24-36.

27 Daugirdas JT, Finn WF, Emmett M, Chertow GM, Frequent Hemodialysis Network Trial G: The phosphate binder equivalent dose. Semin Dial 2011;24:41-49. 